\title{
Commentaire
}

\section{Adaptation et remise à l'épreuve des programmes fondés sur des preuves destinés à prévenir la violence envers les enfants : étude de cas canadienne}

\author{
Christopher Mikton, Ph. D.
}

Diffuser cet article sur Twitter

Ce numéro spécial de Promotion de la santé et prévention des maladies chroniques au Canada est opportun, car la violence envers les enfants constitue un important problème de santé publique : à l'échelle mondiale, des centaines de millions de personnes sont touchées. Un adulte sur quatre dit avoir été victime de violence physique et plus d'un sur trois affirme avoir été victime de violence émotionnelle durant son enfance; 1 femme sur 5 et 1 homme sur 13 indiquent avoir été victimes de violence sexuelle ${ }^{1-3}$. Des enquêtes nationales récentes sur la violence envers les enfants menées en Afrique et dans d'autres pays à revenu faible ou intermédiaire ont fait ressortir des taux de violence physique, sexuelle et émotionnelle encore plus élevés que les taux mondiaux ${ }^{4}$.

Imaginez, propose le $\mathrm{D}^{\mathrm{r}}$ James Mercy, directeur de la Division de la prévention de la violence des Centers for Disease Control and Prevention (CDC) des ÉtatsUnis $^{5}$, qu'un matin, les médias annoncent que des scientifiques ont découvert une nouvelle maladie qui touche jusqu'à 1 milliard d'enfants dans le monde chaque année. Non seulement les enfants exposés à cette maladie sont plus vulnérables à des maladies mentales comme la dépression et les troubles anxieux, à des maladies chroniques comme le diabète, les troubles du cœur et le cancer et à des maladies infectieuses comme l'infection par le VIH, mais ils sont plus nombreux à adopter des comportements antisociaux comme commettre des actes criminels et consommer des drogues. Si une telle maladie existait, que ferions-nous, d'après vous? En fait, cette " maladie » existe déjà : il s’agit de la violence envers les enfants.

Plusieurs organisations, notamment les CDC, l'Organisation mondiale de la santé, le Fonds des Nations unies pour l'enfance et l'Agence de la santé publique du Canada, pour ne nommer qu'elles, ont identifié la prévention de la violence envers les enfants comme priorité mondiale de santé et ont soutenu la prise de mesures de lutte soutenues et efficaces en ce sens.

Il y a toutefois un obstacle important : à l'heure actuelle, peu de méthodes de prévention de la violence envers les enfants se sont avérées efficaces et adaptables à l'échelle d'un pays. Parmi elles, on trouve le Nurse-Family Partnership (NFP) [partenariat entre les infirmières et les familles], programme mis au point aux États-Unis. Les trois essais contrôlés randomisés ayant démontré son efficacité dans la prévention de la violence envers les enfants (réduction de près de moitié) et celle de nombreuses autres conséquences négatives ont également été effectués aux États-Unis.

Comme le met en relief ce numéro spécial ainsi que les publications concernant l'adaptation interculturelle des interventions fondées sur des preuves ${ }^{6-8}$, nous ne pouvons pas affirmer que le NFP mis en œuvre dans de nouveaux contextes obtiendra des résultats aussi impressionnants. Il doit pour cela faire l'objet d'un processus minutieux d'adaptation, de remise à l'épreuve et de perfectionnement pour rester efficace. Le Canada fait des efforts concertés - et exemplaires - en ce sens. Jack et ses collaborateurs $^{9}$ décrivent ainsi le processus rigoureux d'adaptation initiale et d'évaluation de la faisabilité et de l'acceptabilité du NFP en Ontario et en Colombie-Britannique. Li et ses collaborateurs se penchent quant à eux sur les perceptions des professionnels de la santé et des services sociaux qui participent aux aiguillages vers le NFP et qui fournissent les services aux familles inscrites $^{10}$, afin de déterminer comment intégrer le NFP dans un réseau déjà existant de services. Hovdestad et ses collaborateurs $^{11}$ examinent si les enfants de jeunes mères - qui sont le public cible du NFP courent un risque accru d'exposition.

Jack et ses collaborateurs ${ }^{9}$ ont créé un processus d'adaptation et de réévaluation qui va au-delà du modèle en quatre étapes exigé par le $\mathrm{D}^{\mathrm{r}}$ David Olds, concepteur du programme, et son équipe du Centre de recherche sur la prévention de l'Université du Colorado à Denver ${ }^{12}$. Ce processus approfondi, qui comprend une évaluation additionnelle ainsi qu'une étude sur les mécanismes biologiques susceptibles de mettre en évidence la relation entre les interventions et leurs effets sur le

Rattachement de l'auteur :

Prévention de la violence, Gestion des maladies non transmissibles, Prévention des invalidités, de la violence et des blessures, Maladies non transmissibles et santé mentale, Organisation mondiale de la santé, 20, avenue Appia, CH-1211 Genève 27, Suisse

Correspondance : Christopher Mikton, Organisation mondiale de la santé, 20, avenue Appia, CH-1211 Genève 27, Suisse; courriel : miktonc@who.int 
comportement des enfants, se divise en six étapes : 1) adaptation, 2) évaluation de la faisabilité et de l'acceptabilité, 3) essai contrôlé randomisé visant à évaluer l'efficacité, 4) évaluation du processus, 5) étude des mécanismes biologiques potentiels et 6) reproduction et expansion. Cette démarche étant à la fois rigoureuse et soigneusement documentée, notamment par la publication du présent article, elle va pouvoir contribuer à la mise au point d'une méthode empiriquement validée d'adaptation interculturelle et internationale pour les programmes de prévention de la violence envers les enfants ${ }^{13-15}$.

Jack et ses collaborateurs ${ }^{9}$ soulèvent des questions délicates concernant ce processus d'adaptation interculturelle et intercontextuelle et ce processus d'évaluation. Ces questions iront probablement en se complexifiant à mesure que le NFP et des interventions similaires vont être déployés dans des pays aux ressources plus limitées et culturellement distants des pays à l'origine de ces interventions. Dans tous les modèles et cadres d'adaptation interculturelle et intercontextuelle, la fidélité aux éléments essentiels - c'est-à-dire ceux réellement efficaces - de l'intervention est fondamentale pour le maintien de son efficacité dans un nouveau contexte $\mathrm{e}^{7,16-19}$. Ces éléments doivent avoir été identifiés de façon empirique à l'aide de méthodes telles que les essais par répartition en sousgroupes, les analyses de médiation et les micro-essais ${ }^{20}$. On n'a toujours pas établi dans quelle mesure les 18 éléments du modèle établi par le NFP sont efficaces, ni dans quelle mesure ils doivent être respectés pour éviter de compromettre l'efficacité de l'intervention ${ }^{21}$.

L'étude menée par Li et ses collaborateurs ${ }^{10}$ a fait ressortir un élément potentiellement essentiel : la relation individuelle «thérapeutique » à long terme entre l'infirmière ou l'infirmier de santé publique et son client. Cette étude renforce la crédibilité du corpus de données à l'appui du NFP pour les professionnels de la santé publique et des services sociaux, ainsi que la perception selon laquelle le NFP vient combler une lacune majeure même dans un pays riche en ressources comme le Canada. Li et ses collaborateurs ${ }^{10}$ soulèvent la question de savoir dans quelle mesure l'efficacité particulièrement à long terme - du NFP dépend de la qualité des services offerts aux mères avec et après le NFP, ainsi que de leur degré d'intégration. Dans les pays aux ressources limitées, où le personnel infirmier est plus rare, il peut être difficile de maintenir une intervention indépendante dispendieuse comme le NFP. Il est donc d'autant plus important d'en extraire les éléments clés et de les intégrer, à long terme, dans la prestation des services normalement offerts.

En examinant un échantillon de mères de l'Étude canadienne sur l'incidence des signalements de cas de violence et de négligence envers les enfants, Hovdestad et ses collaborateurs ${ }^{11}$ notent que les mères de moins de 22 ans sont associées à des risques plus élevés de mauvaise santé et de problèmes de développement chez l'enfant que les mères plus âgées. Les auteurs ont observé que ces mères étaient plus nombreuses que les autres à présenter certains facteurs de risque modifiables, notamment le fait de bénéficier de l'aide sociale, une consommation abusive d'alcool et une absence de soutien social. Cette étude permet de confirmer empiriquement que les interventions pendant la petite enfance comme le NFP devraient cibler cette population en priorité. Il est toutefois important de vérifier si les jeunes mères présentent aussi des risques plus élevés dans les pays et cultures où les grossesses à un âge plus précoce sont normales, notamment au Bangladesh, en Éthiopie, au Mexique et au Nicaragua, où la proportion de jeunes femmes mères à 18 ans s'élève à respectivement $40 \%, 22 \%, 39 \%$ et $28 \%{ }^{22}$.

Le processus rigoureux d'adaptation et de remise à l'épreuve du NFP au Canada est très prometteur pour la prévention de la violence envers les enfants partout dans le monde. Nous espérons qu'il contribuera à la mise sur pied de méthodes empiriques d'adaptation et de validation internationales et interculturelles. Ces méthodes font cruellement défaut à l'heure actuelle et, sans elles, il est peu probable qu'une intervention fondée sur des preuves puisse être appliquée à l'échelle mondiale pour prévenir cette «maladie» qui affecte des centaines de millions d'enfants dans le monde.

\section{Références}

1. Stoltenborgh M, Bakermans-Kranenburg MJ, Alinik LR, Van IM. The university of childhood emotional abuse: a meta-analysis of worldwide prevalence. J Aggress Maltreat Trauma. 2012;21(8):870-890. DOI: 10.1080/ 10926771.2012.708014.

2. Stoltenborgh M, Bakermans-Kranenburg MJ, Ijzendoorn MJ, Alink LRA. Culturalgeographical differences in the occurrence of child physical abuse? A meta-analysis of global prevalence. Int J Psychol. 2013;48(2): 81-94. DOI: 10.1080/00207594.2012.697165.

3. Stoltenborgh M, Van IM, Euser EM, Bakermans-Kranenburg MJ. A global perspective on child sexual abuse: meta-analysis of prevalence around the world. Child Maltreat. 2011;16(2):79-101. DOI: 10.1177/ 1077559511403920 .

4. Injury Prevention and Control: Division of Violence Prevention. Towards a violencefree generation: using science to fuel action and end violence against children [Internet]. Atlanta (GA) : US Centers for Disease Control and Prevention; 2015 [consulté le $1^{\mathrm{er}}$ mai 2015]. Consultable en ligne à la page : http://www.cdc.gov/violenceprevention/ vacs/index.html

5. Mercy J. CDC measures violence against children, sparks action [Internet]. Atlanta (GA) : CDC Foundation; 25 novembre 2013 [consulté le $1^{\mathrm{er}}$ mai 2015]. Consultable en ligne à la page : http://www.cdcfoundation. org/blog-entry/cdc-measures-violence-againstchildren-sparks-action

6. Barrera M Jr, Castro FG, Steiker LK. Critical analysis of approaches to the development of preventive interventions for subcultural groups. Am J Community Psychol. 2011;48(34):439-454. DOI: 10.1007/s10464-010-9422-X.

7. Cuijpers P, de Graaf I, Bohlmeijer E. Adapting and disseminating effective public health interventions in another country: towards a systematic approach. Eur J Public Health. 2005;15(2):166-169. DOI: 10.1093/ eurpub/cki124. 
8. Office des Nations unies contre la drogue et le crime. Guide d'application des programmes d'acquisition de compétences familiales pour la prévention de l'usage de drogue [Internet]. New York : Nations Unies; février; 2009. PDF téléchargeable à partir du lien : https://www.unodc.org/ documents/prevention/family-guidelinesF.pdf

9. Jack SM, Catherine N, Gonzalez A, MacMillan HL, Sheehan HD, Waddell C, au nom de l'équipe scientifique du British Columbia Healthy Connections Project. Adaptation, mise à l'épreuve et évaluation d'interventions complexes en santé publique : leçons tirées du Nurse-Family Partnership dans le secteur de la santé publique au Canada. Promotion de la santé et prévention des maladies chroniques au Canada. 2015;35(8/9): 161-70.

10. Li SA, Jack SM, Gonzalez A, Duku E, MacMillan H. Points de vue de professionnels de la santé et des services sociaux sur un programme de visites à domicile destiné aux jeunes mères d'un premier enfant. Promotion de la santé et prévention des maladies chroniques au Canada. 2015;35(8/9):171-9.

11. Hovdestad W, Shields M, Williams G, Tonmyr L. Vulnérabilité des familles dirigées par une mère adolescente ou jeune adulte faisant l'objet d'une enquête menée par un service de protection de l'enfance au Canada. Promotion de la santé et prévention des maladies chroniques au Canada. 2015; 35(8/9):152-60.

12. Prevention Research Center for Family and Child Health. Nurse-Family Partnership international program [Internet]. Denver (CO) : University of Colorado Denver; [consulté le 4 décembre 2014].

13. Castro FG, Barrera M Jr, Steiker LKH. Issues and challenges in the design of culturally adapted evidence-based interventions. Annu Rev Clin Psychol. 2010;6:213-239. DOI:10.1146/annurev-clinpsy-033109-132032.

14. Lau AS. Making the case for selective and directed cultural adaptations of evidencebased treatments: Examples from parent training. Clin Psychol-Sci Pr. 2006;13(4):295310. DOI:10.1111/j.1468-2850.2006.00042.x.
15. Mikton C. Two challenges to importing evidence-based child maltreatment prevention programs developed in high-income countries to low-and middle income countries: generalizability and affordability. Dans : H. Dubowitz (dir.), World Perspectives on Child Abuse. (10 éd). Aurora (CO) : International Society for the Prevention of Child Abuse and Neglect; 2012.

16. Falicov CJ. Commentary: On the wisdom and challenges of culturally attuned treatments for Latinos. Fam Process. 2009;48(2):292-309. DOI: 10.1111/j.1545-5300.2009.01282.x.

17. Leerlooijer J, Ruiter RA, Reinders J, Darwisyah W, Kok G, Bartholomew L. The World Starts With Me: using intervention mapping for the systematic adaptation and transfer of schoolbased sexuality education from Uganda to Indonesia. Transl Behav Med. 2011;1(2):33140. DOI:10.1007/s13142-011-0041-3.

18. Wingwood GM, Diclemente RJ. The ADAPT-ITT model: a novel method of adapting evidence-based HIV Interventions. J Acquir Immune Defic Syndr. 2008;47: S40-S46. DOI:10.1097/QAI.0b013e3181605.

19. Kumpfer KL, Pinyuchon M, Teixeiria DMA, Whiteside HO. Cultural adaptation process for international dissemination of the Strengthening Families Program. Eval Health Prof. 2008;31(2):226-239. DOI: 10.1177/ 0163278708315926.

20. Leijten P, Dishion TJ, Thomaes S, Raajimakers MA, de Castro BO, Matthys W. Bringing parenting interventions back to the future: how randomized microtrials may benefit parenting intervention efficacy. Clin Psychol. 2015;22 (1):47-57. DOI: 10.1111/cpsp.12087.

21. Nurse-Family Partnership. Nurse-Family Partnership model elements [Internet]. Denver (CO) : Nurse-Family Partnership; 2011 [consulté le $1^{\text {er }}$ mai 2015]. Consultable en ligne à la page : http://www.nursefamilypartnership. org/communities/model-elements.

22. UNICEF. Satistiques et suivi: Indicateurs économiques par pays [Internet]. New York : UNICEF; 2015 [consultation le $1^{\text {er }}$ mai 2015]; Consultable en ligne à la page : http:// www.unicef.org/french/statistics/index_ countrystats.html 